\title{
A new approach to failure analysis and yield enhancement of very large-scale integrated systems
}

\author{
Shuhei Amakawa \\ Microelectronics Research Centre \\ Cavendish Laboratory \\ Cambridge CB3 OHE, United Kingdom \\ sa264@cam.ac.uk
}

\author{
Kazuo Nakazato and Hiroshi Mizuta \\ Hitachi Cambridge Laboratory \\ Cavendish Laboratory \\ Cambridge CB3 OHE, United Kingdom \\ nakazato@phy.cam.ac.uk
}

\begin{abstract}
A method of failure analysis is developed based on probability theory. Unlike Monte Carlo methods, it produces accurate results even when the probabilities of interest differ from one another by many orders of magnitude. The method is applied to the analysis of the leakagecurrent distribution of double-gate MOSFETs and the microscopic failure mechanism is identified that limits the final yield. It explains experimental data very well. The insight into the failure mechanism gives clear guidelines for yield enhancement and facilitates device design together with the quantitative yield prediction.
\end{abstract}

\section{Introduction}

Development of very large-scale integrated systems (VLSIs) has been spurred continuously in the past decades. Since it is usually not possible to repair faulty component devices in a VLSI, each device in a chip can become a single point of failure unless some redundancy is introduced. Therefore, VLSIs have to be designed based on the characteristics of worst devices rather than those of average devices. Even if a chip is equipped with some redundant devices, today's scale of integration is becoming so high that the yield requirement is still very severe. For instance, a 1 Gbit DRAM with $10^{3}$ redundant cells would require the device yield of at least $99.9999 \%$. The final chip yield is governed by the device yield.

Once the major cause of failure is somehow identified or assumed, one could use a Monte Carlo method to study yield problems. This is a very common approach. One serious practical limitation of Monte Carlo methods is that events of interest (failures) must occur very many times for the result to make any quantitative sense. However, if failure probabilities are very low, most of the computation time is consumed by uninteresting events, without producing much useful result. To cope with this, we develop using probability theory a framework for calculating the probability distribution of stochastic events whose probabilities may be extremely low. Although our approach is fairly general and may have various applications, we shall illustrate it by means of a concrete example: probability

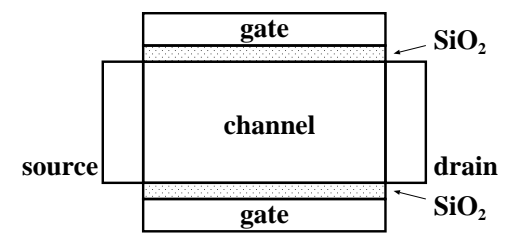

Figure 1. A schematic cross sectional view of a doublegate MOSFET.

distribution of leakage current in double-gate (DG) MOSFETs (Fig. 1).

\section{The problem defined}

DG MOSFETs are expected to scale well below $0.1 \mu \mathrm{m}[1,2]$. Our DG MOSFETs have poly-Si channels and were fabricated with a $0.2 \mu \mathrm{m}$ process technology. The distribution of measured leakage currents in the off state is plotted in Fig. 2 as dots. The device yield is given by the cumulative probability at a given tolerable leakage current. Most of the DG MOSFETs had ideal characteristics, but some of them exhibited very large leakage currents as shown in the graph. The measured leakage current increases rapidly above the cumulative probability of around $80 \%$. This part of the distribution is known as the tail distribution and has actively been studied in the context of DRAM retention time [3-7].

Characteristics of the average devices, those in the main distribution, were adequately explained by conventional two-dimensional device simulation. In an attempt to explain the characteristics of the faulty devices in the tail distribution, we modified a two-dimensional device simulator to include a generic model of fieldenhanced thermally-assisted trap-to-band tunnelling proposed by Lui and Migliorato [8]. This model unifies major mechanisms of carrier generation and recombination mediated by traps: the SRH generation-recombination [9], Poole-Frenkel barrier lowering, and thermally-assisted tunnelling. It turned out that the trap model provides a very good account of the leakage-current characteristics. Thus we concluded that the field-enhanced carrier generation by traps is the main cause of the failure. We now want to reproduce the experimental leakage-current distribution 


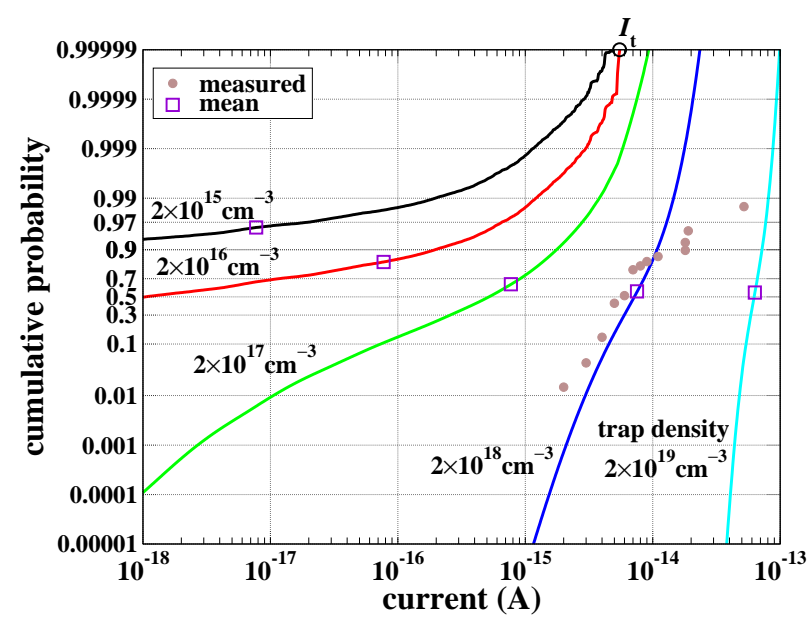

Figure 2. Cumulative probability distributions of leakage currents in DG MOSFETs in the off state. The filled dots are the experimental data. The solid lines are the calculated distributions. The open squares represent the mean currents. The total trap density is varied as a parameter while keeping the shape of the trap level distribution (Fig. 3) unchanged. The leakage current at the cumulative probability of $\sim 99.9999 \%$ is bounded by $I_{\mathrm{t}}$, the maximum current generated by a single trap.

by modelling and predict how the yield changes depending on various factors, of which some are controllable.

\section{Calculation procedure}

First, the device is meshed and a two-dimensional device simulation is run without incorporating the new trap model. This is to calculate the leakage current due to other mechanisms (mainly diffusion) and also to obtain the electric field and electron and hole densities, which are to be used to calculate the trap-generated current later. The implicit assumption here is that the presence of traps does not affect the electric field and carrier densities very much.

Next the computation of the distribution of trapgenerated currents follows. We assume a spatially uniform trap distribution in the channel. Since the channel is poly-Si, traps take continuous energy levels within the energy gap [8]. A trap is either donor type or acceptor type. We use the trap level distribution shown in Fig. 3. The electric field and the carrier densities are assumed to be constant within a finite element. Given the electric field, carrier densities, the trap type and the trap level, the current generated by a trap in the finite element can be calculated using Lui and Migliorato's formula [8]. The total trap-generated current in the device, $I_{\text {trap }}$, is given by the sum of such currents.

The leakage current differs from device to device because each device has different number of traps of either type at different sites at different energy levels. The probability to find a certain trap configuration can be calculated if the total trap density is specified. The leakage current

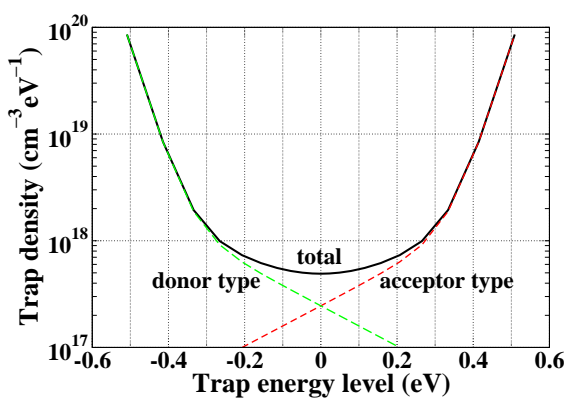

Figure 3. A trap level distribution used in the calculation [8], which models poly-silicon. The total trap density is $2 \times 10^{18} \mathrm{~cm}^{-3}$ in the case shown above. The origin of the abscissa is the mid-gap of silicon.

for that trap configuration can also be calculated as explained above. Therefore, we can compute the probability density function $\rho\left(I_{\text {trap }}\right)$ of the trap-generated current by taking into account all possible trap configurations.

Let $U_{m}$ be the volume of the $m$ th finite element. The mean trap number in it is $\langle n\rangle=U_{m} N_{\mathrm{T}}$, where $N_{\mathrm{T}}=$ $\sum_{j} N_{\mathrm{T} j}$ is the total trap density. $N_{\mathrm{T} j}$ is the density of traps at the $j$ th energy level $E_{\mathrm{T} j}$. $N_{\mathrm{T} j}$ is given according to Fig. 3. For notational simplicity, let $j$ index not only the energy level but also the trap type. The probability to find $n$ traps in the $m$ th element can be approximated to the Poisson distribution [10] $P_{m}(n)=\langle n\rangle^{n} \mathrm{e}^{-\langle n\rangle} / n$ !. If there are $n$ traps in the $m$ th finite element, the probability of $i$ of them being at $E_{\mathrm{T} j}$ is given by a binomial distribution. Therefore, the probability of finding $n$ traps in the $m$ th element and $i$ of them being at $E_{\mathrm{T} j}$ is

$$
P_{m j}(n, i)=P_{m}(n)\left(\begin{array}{c}
n \\
i
\end{array}\right)\left(\frac{N_{\mathrm{T} j}}{N_{\mathrm{T}}}\right)^{i}\left(1-\frac{N_{\mathrm{T} j}}{N_{\mathrm{T}}}\right)^{n-i} .
$$

The probability of having $i$ traps at $E_{\mathrm{T} j}$ in the $m$ th element is

$$
P_{m j}(i)=\sum_{n=i}^{R_{m}} P_{m j}(n, i),
$$

where $R_{m}$ is a number chosen such that $\langle n\rangle / R_{m} \ll 1$. $P_{m j}(i)$ satisfies $\sum_{i=0}^{R_{m}} P_{m j}(i)=1$. Let $\Delta I_{m j}$ denote the current generated by a trap at $E_{\mathrm{T} j}$ in the $m$ th finite element. Then $i \Delta I_{m j}$ will be generated by $i$ such traps with the probability $P_{m j}(i) . \quad I_{\text {trap }}$ is the sum of $i \Delta I_{m j}$ over $j, i$ and $m$. Evidently, the functional relationship between $I_{\text {trap }}$ and the probability density is implicit, but can be computed numerically by iteration. The calculation procedure is summarised in Fig. 4. We would like to emphasise that the traps are treated as discrete, countable entities. This discreteness will turn out very important.

\section{Results and discussion}

Calculated leakage-current distributions (i.e. the integrals of the probability density functions) are shown in Fig. 2 for several trap densities. One of the results agree 


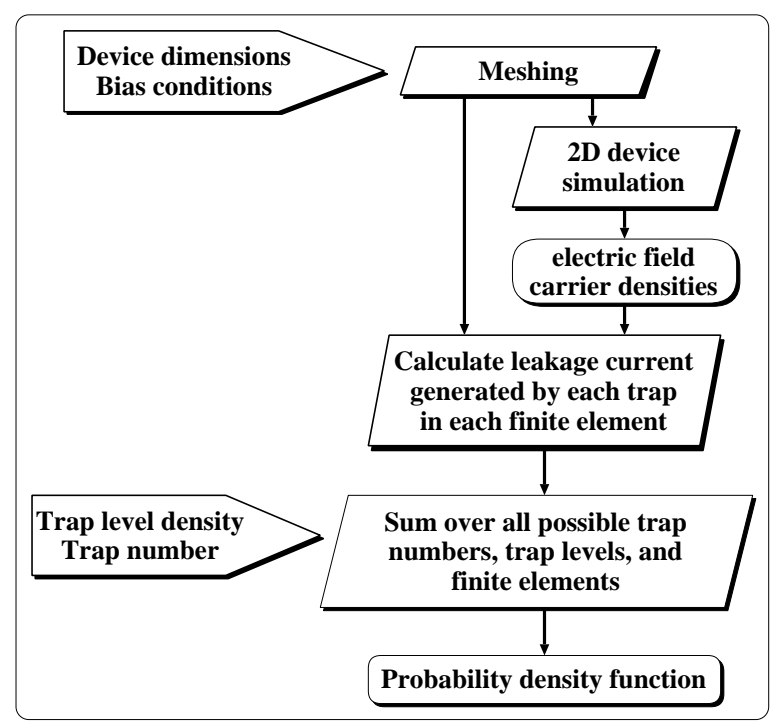

Figure 4. The calculation procedure of the trapgenerated current distribution. First, the electric field and the carrier densities are calculated by a device simulator. In each finite element, the current generated by one trap is calculated for each trap level and trap type. Probabilities and currents are summed over all possible trap configurations.

well with the experimental data in the main region. We shall put aside the discrepancy in the tail region for the moment and look at the calculated results.

The calculated tail currents (at the cumulative probability of, say, $99.9999 \%$ ) are bounded by a certain value $I_{\mathrm{t}}$, even when the trap density is very low (Fig. 5). Figure 6 shows the maximum possible current generated by one trap, $\max _{j}\left(\Delta I_{m j}\right)$, in each finite element $m$. Very large currents are generated in the high-field regions near the drain. The peak current, $\max _{j, m}\left(\Delta I_{m j}\right)$, in Fig. 6 is the $I_{\mathrm{t}}$ that appeared in Fig. 2 as the lower bound. This is not a coincidence. There always is a finite probability to have a trap that generates $I_{\mathrm{t}}$, however low the trap density may be. Since traps are treated as discrete entities, $I_{\mathrm{t}}$ appears at the probability tail, setting the lower bound. If some traps happen to exist in the high-field regions at some undesirable energy levels (near the mid-gap), the transistor fails [5]. If $I_{\mathrm{t}}$ is larger than the tolerable leakage current, even a single trap will lead to the failure of a transistor.

More work is needed to reproduce the tail distribution experimentally observed. One thing that is missing in our modelling is the spatial non-uniformity of the trap distribution. Traps may actually be localised to grain boundaries of poly-Si. We were, however, unable to reproduce the tail distribution by introducing such spatial nonuniformities. After all, the tail distribution exists also in the case of single-crystal MOSFETs in which trap distribution should be more uniform.

Another possibility to look at is the fluctuation of the electric field within a device. So far we have used the same field for all trap configurations. But the potential profile

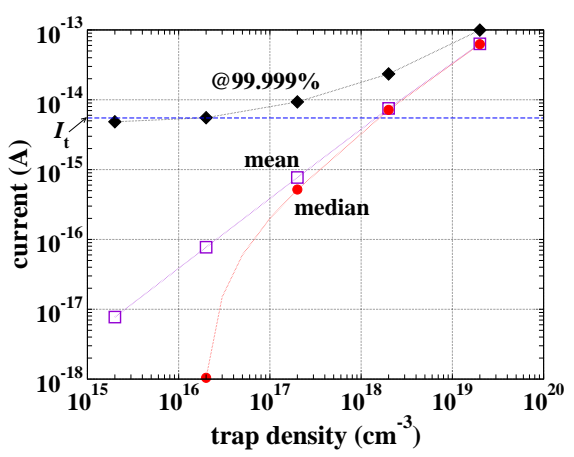

Figure 5. The mean current, the median current, and the tail current at the cumulative probability of $99.999 \%$ versus total trap density. $I_{\mathrm{t}}=\max _{j, m}\left(\Delta I_{m j}\right)$.

in each device may differ slightly from others' for many reasons, such as existence of defects and grain boundaries. Then, we note that a slight difference in a local potential profile can result in a significant difference in its slope (electric field), especially when the slope is steep there. Because of poly-Si grain boundaries, there should be much greater potential fluctuation in our devices than in single-crystal MOSFETs. Thus some ad hoc local field enhancement is added in some areas, with altering the calculation procedure accordingly. A similar model was proposed by Hiraiwa et al. [5]. The enhanced electric field raises $I_{\mathrm{t}}$ a great deal because of the field-induced barrier lowering [8], and gives rise to the tail distribution as shown in Fig. 7. The amount of field enhancement has a far greater effect on the tail current than the area in which the field is enhanced because only the former affects $I_{\mathrm{t}}$.

Reduction of the trap density has little effect on the tail current if the mean current is less than $I_{\mathrm{t}}$ (Fig. 5). What is truly effective is to reduce $I_{\mathrm{t}}$ itself. The important point here is the sensitivity of $I_{\mathrm{t}}$ to the electric field. As an example of yield enhancing design, DG MOSFETs with additional lightly-doped drain and source regions are simulated. The highest field near the drain should become lower if properly designed, thereby leading to a lower $I_{\mathrm{t}}$ and a better yield. Figure 8 shows that an optimum donor concentration exists that minimises the tail current. Other examples may include the use of thicker gate oxide near the drain. Improvement of the channel quality would also be effective, but this would be primarily due to the reduction of the electric field fluctuation rather than the reduction in the trap number.

In conclusion, we successfully described the distribution of leakage currents in DG MOSFETs starting from a microscopic model. We showed that $I_{\mathrm{t}}$, the maximum possible current generated by a single trap, governs the device yield. Our method proved to be useful for yield prediction and device design. Transistors should be designed such that $I_{\mathrm{t}}$ is very much lower than the tolerable leakage current at the specified cumulative probability. Our method does not have any convergence problems as in the conventional Monte Carlo approach. 


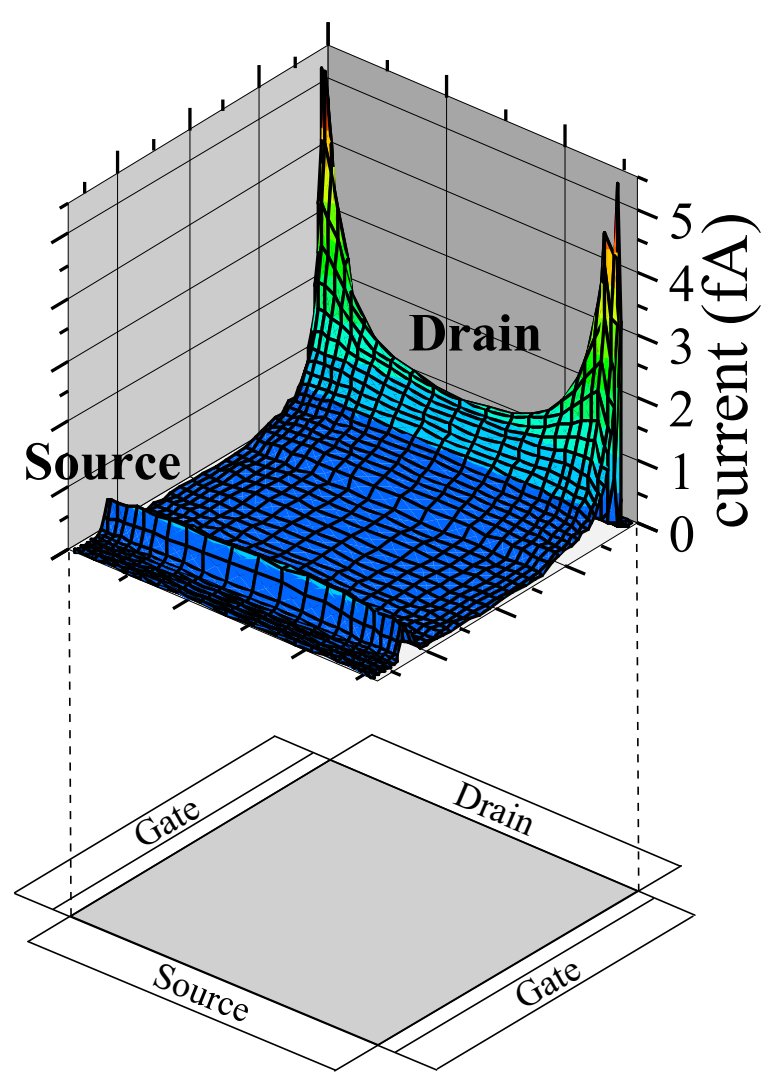

Figure 6. The maximum current that can be generated by a single trap in the off state, as a function of the trap position. The current peaks occur near the drain $\mathrm{n}^{+}$$\mathrm{p}^{-}$junction where the electric field becomes very high. The peak current value, referred to as $I_{\mathrm{t}}$, appears in Fig. 2. The simulated channel length is $0.35 \mu \mathrm{m}$ and the channel width is $0.13 \mu \mathrm{m}$. $V_{\mathrm{DS}}=1.8 \mathrm{~V}, V_{\mathrm{GS}}=-0.5 \mathrm{~V}$.

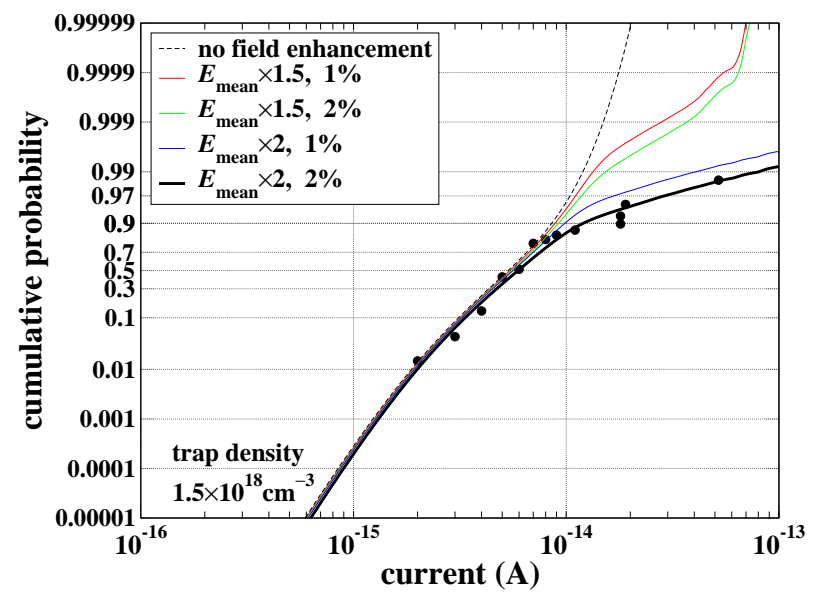

Figure 7. Cumulative probability distributions of leakage currents in DG MOSFETs with local electric field fluctuation. The electric field is enhanced by some factor in some areas. $E_{\text {mean }}$ is the field calculated by the device simulator. The dots are the experimental data.

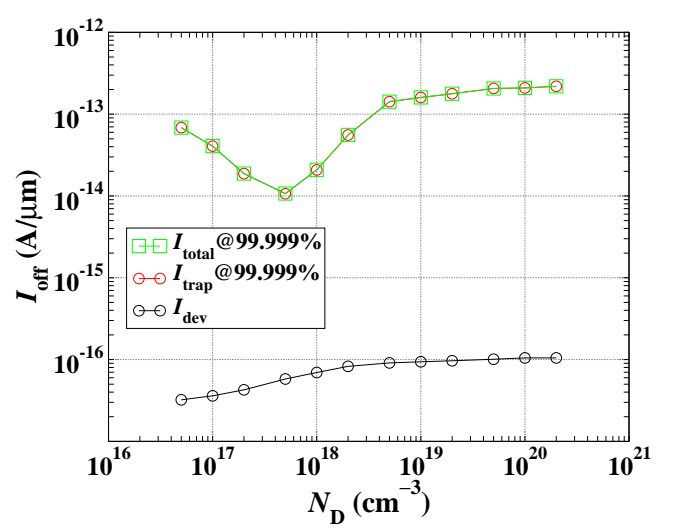

Figure 8. Dependence of the tail current on the donor concentration in the lowly-doped drain and source regions. $I_{\text {trap }}$ is the trap-generated leakage current. $I_{\mathrm{dev}}$ is the leakage current calculated by the device simulator. $I_{\text {total }}=I_{\text {trap }}+I_{\mathrm{dev}}$. The lowly-doped regions are $50 \mathrm{~nm}$ in length each.

[1] K. Suzuki, T. Tanaka, Y. Tosaka, H. Horie and Y. Arimoto, "Scaling theory for double-gate SOI MOSFET's," IEEE Trans. Electron Devices, 40, pp. 2326-2329, 1993.

[2] S.-H. Oh, D. Monroe and J. M. Hergenrother, "Analytic description of short-channel effects in fully-depleted doublegate and cylindrical, surrounding-gate MOSFETs," IEEE Electron Device Lett., 21, pp. 445-447, 2000.

[3] A. Hiraiwa, M. Ogasawara, N. Natsuaki, Y. Itoh and H. Iwai, "Field-effect trap-level-distribution model of dynamic random access memory data retention characteristics,” J. Appl. Phys., 81, pp. 7053-7060, 1997.

[4] T. Hamamoto, S. Sugiura and S. Sawada, "On the retention time distribution of dynamic random access memory (DRAM)," IEEE Trans. Electron Devices, 45, pp. 13001309, 1998.

[5] A. Hiraiwa, M. Ogasawara, N. Natsuaki, Y. Itoh and H. Iwai, "Local-field-enhancement model of DRAM retention failure," Tech. Digest Int. Electron Devices Meeting, pp. 157-160, 1998.

[6] S. Ueno, Y. Inoue, M. Inuishi and N. Tsubouchi, "Leakage mechanism of local junctions forming the main or tail mode of retention characteristics for dynamic random access memories," Jpn. J. Appl. Phys., 39, pp. 1963-1968, 2000.

[7] K. Yamaguchi, "Theoretical study of deep-trap-assisted anomalous currents in worst-bit cells of dynamic randomaccess memories (DRAM's)," IEEE Trans. Electron Devices, 47, pp. 774-780, 2000.

[8] O. K. B. Lui and P. Migliorato, "A new generationrecombination model for device simulation including the Poole-Frenkel effect and phonon-assisted tunnelling," Solid-State Electron., 41, pp. 575-583, 1997.

[9] W. Shockley and W. T. Read, Jr., "Statistics of the recombinations of holes and electrons," Phys. Rev., 87, pp. 835$842,1952$.

[10] W. Shockley, "Problems related to p-n junctions in silicon," Solid-State Electron., 2, pp. 35-67, 1961. 